\title{
SHORT WAVE STABILITY FOR INVISCID SHEAR FLOW*
}

\author{
MICHAEL RENARDY ${ }^{\dagger}$
}

\begin{abstract}
We consider the linear stability of inviscid shear flows. While it is well known that discontinuous velocity profiles lead to short wave instabilities and ill-posedness, known examples of instability for smooth profiles have a short wave cutoff; i.e., there is a critical wave number beyond which no unstable eigenvalues exist. This paper proves a result to this effect under suitable assumptions on the base flow profile.
\end{abstract}

Key words. hydrodynamic stability, Rayleigh equation, inviscid shear flow

AMS subject classification. 76E05

DOI. $10.1137 / 080720905$

1. Introduction. The linear stability of inviscid shear flows has been studied extensively over the past century. I refer to $[1,2,3]$ for reviews. Discontinuous velocity profiles lead to the Kelvin-Helmholtz instability which has unbounded growth rates in the limit of high wave numbers. In contrast, the instabilities in shear flows with smooth velocity profiles appear to be long wave instabilities. That is, there is cutoff at some maximal wave number $\alpha_{m}$ such that there are no unstable eigenvalues for $\alpha>\alpha_{m}$.

Although all known examples appear to satisfy this, I have not been able to find a proof in the literature. Almost half a century ago, Howard [4] proved such a result for a special case. He assumes that all inflection points of the base flow profile $U$ occur at the same value $U_{i}$ and that $U^{\prime \prime} /\left(U-U_{i}\right)$ is bounded and of one sign.

The objective of this paper is to prove the nonexistence of unstable eigenvalues for large $\alpha$ in more general velocity fields. In the proof, it turns out that the crucial difficulty for the analysis occurs at critical points of $U$ rather than inflection points. A critical point of $U$ is a point where $U^{\prime}=0 .^{1}$

We shall need the following assumptions:

1. All critical points are isolated. Moreover, in a neighborhood of each critical point $y_{c}, U^{\prime \prime}(y)\left(U(y)-U\left(y_{c}\right)\right)$ is nonnegative.

2. If $y_{c}$ is a critical point, and $U(y)=U\left(y_{c}\right)$, then $y$ is also a critical point.

The first assumption is satisfied for all analytic velocity profiles. I suspect that the second assumption is not necessary, but I do not know how to avoid it in the proof. Even if the second assumption does not hold, it will be shown that growth rates of unstable modes must approach zero at an exponential rate as $\alpha \rightarrow \infty$.

2. Proof of short wave stability. Our goal is the following result.

THEOREM 2.1. Let $U(y)$ be an analytic function defined for $y \in[0,1]$. Moreover, assume $U$ has the following property: If $U^{\prime}\left(y_{0}\right)=0$ for some $y_{0} \in[0,1]$, then $U^{\prime}(y)=0$

*Received by the editors April 10, 2008; accepted for publication (in revised form) September 9, 2008; published electronically December 17, 2008. This research was supported by the National Science Foundation under grant DMS-0707727.

http://www.siam.org/journals/siap/69-3/72090.html $\mathrm{edu})$.

$\dagger$ Department of Mathematics, Virginia Tech, Blacksburg, VA 24061-0123 (renardym@math.vt.

${ }^{1}$ This usage of the word "critical", which is common in calculus of variations, should not be confused with a totally unrelated usage of the same word, which also occurs in hydrodynamic stability studies. 
for every $y$ with $U(y)=U\left(y_{0}\right)$. Then there exists $\alpha_{0}$ such that, for $\alpha>\alpha_{0}$, all eigenvalues $c$ of the Rayleigh equation (equation (21.17) in [2]),

$$
(U(y)-c)\left(\psi^{\prime \prime}(y)-\alpha^{2} \psi(y)\right)-U^{\prime \prime}(y) \psi(y)=0,
$$

with boundary condition $\psi(0)=\psi(1)=0$ are real.

The assumptions allow, for instance, monotone velocity profiles, profiles with a single maximum or minimum, and periodic profiles with one maximum and minimum.

For the proof, let us assume $c$ is an eigenvalue which is not real. We can write the equation in the form

$$
\psi^{\prime \prime}-\alpha^{2} \psi=\frac{U^{\prime \prime}}{U-c} \psi
$$

We multiply by the conjugate of $\psi$ and integrate, which yields

$$
\int_{0}^{1}\left|\psi^{\prime}\right|^{2}+\alpha^{2}|\psi|^{2} d y=-\int_{0}^{1} \frac{U^{\prime \prime}(y)(U(y)-\bar{c})}{|U(y)-c|^{2}}|\psi|^{2} d y
$$

With $c=c_{r}+i c_{i}$, this yields the two separate equations

$$
\begin{aligned}
\int_{0}^{1}\left|\psi^{\prime}\right|^{2}+\alpha^{2}|\psi|^{2} d y & =-\int_{0}^{1} \frac{U^{\prime \prime}(y)\left(U(y)-c_{r}\right)}{\left(U(y)-c_{r}\right)^{2}+c_{i}^{2}}|\psi|^{2} d y, \\
0 & =\int_{0}^{1} \frac{U^{\prime \prime}(y)}{\left(U(y)-c_{r}\right)^{2}+c_{i}^{2}}|\psi|^{2} d y .
\end{aligned}
$$

The overall strategy of the proof is to show that, for large $\alpha$, contributions to the right-hand side of the first equation in (2.4) are either negative or not large enough to balance the left-hand side. Clearly, we have

$$
\left|\frac{U^{\prime \prime}(y)\left(U(y)-c_{r}\right)}{\left(U(y)-c_{r}\right)^{2}+c_{i}^{2}}\right| \ll \alpha^{2}
$$

unless $U(y)-c_{r}$ is small of order $\alpha^{-2}$. To focus the discussion further, we divide the points in $[0,1]$ into the following two categories:

1. $y$ is a regular point if $U^{\prime}(y) \neq 0$.

2. $y$ is a critical point if $U^{\prime}(y)=0$.

Since we assumed $U$ to be analytic, all but finitely many points are regular. We shall call $y$ a $\delta$-regular point if the distance from the nearest critical point is at least $\delta$.

The next lemma will be used repeatedly in what follows.

Lemma 2.2. Let $x_{0} \in[0,1]$, and for given $u \in L^{2}[0,1]$, let

$$
L u(x)=\frac{1}{x-x_{0}} \int_{x_{0}}^{x} u(\xi) d \xi .
$$

Then the operator $L$ is a bounded mapping from $L^{2}[0,1]$ into itself.

This result follows immediately from Theorem 11.8 in [5], but for the sake of keeping this paper self-contained, I shall give the proof.

Let $v(x)=L u(x)$. We have $u=\left(\left(x-x_{0}\right) v\right)^{\prime}$, and hence $2 u v-v^{2}=\left(\left(x-x_{0}\right) v^{2}\right)^{\prime}$ (in particular, this implies that $\left(x-x_{0}\right) v^{2}$ is continuous, since $2 u v-v^{2}$ is integrable). Consequently,

$$
\int_{0}^{1} 2 u v d x=\left(1-x_{0}\right) v(1)^{2}+x_{0} v(0)^{2}+\int_{0}^{1} v^{2} d x
$$

Copyright $@$ by SIAM. Unauthorized reproduction of this article is prohibited. 
which implies $\|v\| \leq 2\|u\|$. Here and in what follows, $\|\cdot\|$ refers to the norm in $L^{2}(0,1)$.

We shall begin with an estimate for regular points.

Lemma 2.3. Let $z$ be a $2 \delta$-regular point, and let $c_{r}=U(\tilde{z})$ where $|z-\tilde{z}|<\delta / 2$. Then there is a constant $C$, depending on $\delta$ but not on $z$ and $c$, such that

$$
\left.\left|\int_{z-\delta}^{z+\delta} \frac{U^{\prime \prime}(y)\left(U(y)-c_{r}\right)}{\left(U(y)-c_{r}\right)^{2}+c_{i}^{2}}\right| \psi\right|^{2} d y \mid \leq C\left(\|\psi\|^{2}+\|\psi\|^{1 / 2}\left\|\psi^{\prime}\right\|^{3 / 2}\right) .
$$

If $z$ is close to the boundary, the interval $[z-\delta, z+\delta]$ may not be contained in $[0,1]$. In this case, however, we can simply extend $\psi$ by zero outside of $[0,1]$. Due to the boundary condition $\psi(0)=\psi(1)=0$, this continuation is still in $H^{1}$.

Clearly, there is a lower bound for $U^{\prime}$ on the set of all $\delta$-regular points. Let $q(\delta)$ be this lower bound. Then we have $\left|U(y)-c_{r}\right| \geq q(\delta) \delta / 2$ if $|y-\tilde{z}|>\delta / 2$. In this range of $y$, we can therefore estimate the integrand by

$$
\frac{2 \max \left|U^{\prime \prime}\right|}{\delta q(\delta)}|\psi|^{2}
$$

Hence we only need to concern ourselves with

$$
\int_{\tilde{z}-\delta / 2}^{\tilde{z}+\delta / 2} \frac{U^{\prime \prime}(y)\left(U(y)-c_{r}\right)}{\left(U(y)-c_{r}\right)^{2}+c_{i}^{2}}|\psi|^{2} d y
$$

In this integral, we set

$$
\psi(y)=\psi(\tilde{z})+(y-\tilde{z}) \chi(y)
$$

and

$$
|\psi(y)|^{2}=|\psi(\tilde{z})|^{2}+(y-\tilde{z})(\psi(y) \bar{\chi}(y)+\chi(y) \bar{\psi}(\tilde{z})) .
$$

According to Lemma 2.2 above, we have $\|\chi\| \leq 2\left\|\psi^{\prime}\right\|$, and from the trace theorem (see (3.18) in [5]) we have $|\psi(\tilde{z})|^{2} \leq C\left(\|\psi\|^{2}+\|\psi\|\left\|\psi^{\prime}\right\|\right)$ for some constant $C$. Moreover,

$$
\frac{U^{\prime \prime}(y)\left(U(y)-c_{r}\right)(y-\tilde{z})}{\left(U(y)-c_{r}\right)^{2}+c_{i}^{2}}
$$

is bounded. Consequently, we find

$$
\begin{aligned}
& \mid \int_{\tilde{z}-\delta / 2}^{\tilde{z}+\delta / 2} \frac{U^{\prime \prime}(y)\left(U(y)-c_{r}\right)(y-\tilde{z})}{\left(U(y)-c_{r}\right)^{2}+c_{i}^{2}}(\psi(y) \bar{\chi}(y)+\chi(y) \bar{\psi}(\tilde{z})) d y \mid \\
& \leq C(\delta)\left(\|\psi\|\|\chi\|+|\psi(\tilde{z})|\|\chi\| \leq C(\delta)\left(\|\psi\|^{2}+\left\|\psi^{\prime}\right\|^{3 / 2}\|\psi\|^{1 / 2}\right) .\right.
\end{aligned}
$$

It remains to estimate

$$
|\psi(\tilde{z})|^{2}\left|\int_{\tilde{z}-\delta / 2}^{\tilde{z}+\delta / 2} \frac{U^{\prime \prime}(y)\left(U(y)-c_{r}\right)}{\left(U(y)-c_{r}\right)^{2}+c_{i}^{2}} d y\right| .
$$

In this last integral, we substitute $U(y)$ as a new variable to obtain the new integral

$$
\int_{U(\tilde{z}-\delta / 2)}^{U(\tilde{z}+\delta / 2)} \frac{U^{\prime \prime}(y(U))\left(U-c_{r}\right)}{U^{\prime}(y(U))\left[\left(U-c_{r}\right)^{2}+c_{i}^{2}\right]} d U .
$$

Copyright $@$ by SIAM. Unauthorized reproduction of this article is prohibited. 
The interval of integration contains the symmetric interval $\left|U-c_{r}\right|<\delta q(\delta) / 2$, and outside this interval, the integrand is bounded by a constant $C(\delta)$. Next, we write

$$
\frac{U^{\prime \prime}(y(U))}{U^{\prime}(y(U))}=\frac{U^{\prime \prime}(\tilde{z})}{U^{\prime}(\tilde{z})}+\left(U-c_{r}\right) S(U)
$$

where $S(U)$ is a continuous function. By symmetry, we then find

$$
\int_{c_{r}-\delta q(\delta) / 2}^{c_{r}+\delta q(\delta) / 2} \frac{U^{\prime \prime}(y(U))\left(U-c_{r}\right)}{U^{\prime}(y(U))\left[\left(U-c_{r}\right)^{2}+c_{i}^{2}\right]} d U=\int_{c_{r}-\delta q(\delta) / 2}^{c_{r}+\delta q(\delta) / 2} \frac{\left(U-c_{r}\right)^{2} S(U)}{\left(U-c_{r}\right)^{2}+c_{i}^{2}} d U .
$$

The integrand in the latter integral is bounded by a constant. This completes the proof of Lemma 2.3.

We next consider the neighborhood of a critical point.

Lemma 2.4. There exist $\epsilon>0$ and $K>0$ such that, if $y_{0}$ is in an $\epsilon$-neighborhood of a critical point $y_{c}$, and $c_{r}=U\left(y_{0}\right)$, then

$$
\int_{0}^{1} \frac{U^{\prime \prime}(y)\left(U(y)-c_{r}\right)}{\left(U(y)-c_{r}\right)^{2}+c_{i}^{2}}|\psi|^{2} d y \geq-K\|\psi\|^{2} .
$$

We exploit the second equation of (2.4) to obtain that

$$
\int_{0}^{1} \frac{U^{\prime \prime}(y)\left(U(y)-c_{r}\right)}{\left(U(y)-c_{r}\right)^{2}+c_{i}^{2}}|\psi|^{2} d y=\int_{0}^{1} \frac{U^{\prime \prime}(y)\left(U(y)-U\left(y_{c}\right)\right)}{\left(U(y)-c_{r}\right)^{2}+c_{i}^{2}}|\psi|^{2} d y .
$$

All values of $y$ where $U(y)=U\left(y_{c}\right)$ are critical points, and there is a finite number of these. Each of them has a neighborhood on which $U^{\prime \prime}(y)\left(U(y)-U\left(y_{c}\right)\right)$ is nonnegative. If we choose $\epsilon$ small enough, then $\left|U(y)-c_{r}\right|$ has a positive lower bound outside these neighborhoods. The lemma follows.

To prove the theorem, we need to bound the right-hand side in the first equation of (2.4). Lemma 2.3 gives a bound of the form

$$
C\left(\|\psi\|^{2}+\|\psi\|^{1 / 2}\left\|\psi^{\prime}\right\|^{3 / 2}\right)
$$

in the neighborhood of regular points. Lemma 2.4 gives an upper bound of the form $K\|\psi\|^{2}$ in the neighborhood of critical points. By combining the two, we find that the right-hand side in the first equation of (2.4) cannot balance the left-hand side if $\alpha$ is large and $\psi$ is nontrivial, which is the desired result.

We note that the assumption of analyticity was used only to ensure that the number of critical points is finite and that $\left(U(y)-U\left(y_{c}\right)\right) U^{\prime \prime}(y)$ is nonnegative in a neighborhood of each critical point.

Without the assumption that $U$ assumes its critical values only at critical points, we can still prove that the growth rate of unstable modes must tend to zero at an exponential rate as $\alpha \rightarrow \infty$.

TheOrem 2.5. Let $U$ be any analytic function on $[0,1]$. Then there exists a function $s(\alpha)$, with $s(\alpha) \rightarrow 0$ for $\alpha \rightarrow \infty$, such that, if $c$ is a nonreal eigenvalue of the Rayleigh equation, and $\alpha$ is sufficiently large, then there exists a critical point $y_{c}$ with $\left|c-U\left(y_{c}\right)\right| \leq s(\alpha)$. Moreover, there exist constants $C$ and $k$ such that $\left|\alpha c_{i}\right| \leq$ $C\left|c-U\left(y_{c}\right)\right| \exp (-k|\alpha|)$.

The first statement, that $c_{r}$ must be close to a critical value of $U$ when $\alpha$ is large, follows from the proof of the previous theorem. Now let $\rho$ be a nonnegative function 
which vanishes in an $\epsilon$-neighborhood of the critical points but is equal to 1 at distance more than $2 \epsilon$ from the critical points. We multiply the Rayleigh equation by $\rho \bar{\psi}$ and integrate. The result is

$$
\int_{0}^{1} \rho\left(\left|\psi^{\prime}\right|^{2}+\alpha^{2}|\psi|^{2}\right) d y=-\int_{0}^{1} \frac{U^{\prime \prime}(y)(U(y)-\bar{c})}{|U(y)-c|^{2}} \rho|\psi|^{2} d y-\int_{0}^{1} \rho^{\prime} \psi^{\prime} \bar{\psi} d y .
$$

With the help of Lemma 2.3, this yields the estimate

$$
\int_{0}^{1} \rho\left(\left|\psi^{\prime}\right|^{2}+\alpha^{2}|\psi|^{2}\right) d y \leq C\left(\|\psi\|^{2}+\|\psi\|^{1 / 2}\left\|\psi^{\prime}\right\|^{3 / 2}\right) .
$$

Now let $y_{c}$ be a critical point, and let $\chi$ be a smooth function which is equal to 1 in an $\epsilon$-neighborhood of $y_{c}$ and has support in a $2 \epsilon$-neighborhood of $y_{c}$. We can choose $\epsilon$ such that $U^{\prime \prime}\left(U-U\left(y_{c}\right)\right)$ is nonnegative on the support of $\chi$. We shall also assume that $c_{r}=U\left(y_{1}\right)$, where $y_{1}$ is within $\epsilon / 2$ of $y_{c}$. We multiply the Rayleigh equation by $\chi \bar{\psi}$ and integrate. The result is

$$
\int_{0}^{1} \chi\left(\left|\psi^{\prime}\right|^{2}+\alpha^{2}|\psi|^{2}\right) d y=-\int_{0}^{1} \frac{U^{\prime \prime}(y)(U(y)-\bar{c})}{|U(y)-c|^{2}} \chi|\psi|^{2} d y-\int_{0}^{1} \chi^{\prime} \psi^{\prime} \bar{\psi} d y .
$$

Taking the imaginary part of this identity, we conclude that

$$
\left.\left|c_{i}\right|\left|\int_{0}^{1} \frac{U^{\prime \prime}}{|U-c|^{2}} \chi\right| \psi\right|^{2} d y|\leq| \int_{0}^{1} \chi^{\prime} \psi^{\prime} \bar{\psi} d y \mid .
$$

For large $\alpha$, the solutions of the Rayleigh equation have exponential asymptotics as long as we stay away from points where $U-c$ is small (see Theorem 26.3 in [6]). Since the support of $\chi^{\prime}$ is separated from the points where $|U-c|$ is small, we can conclude that there is a bound of the form

$$
\left|\int_{0}^{1} \chi^{\prime} \psi^{\prime} \bar{\psi} d y\right| \leq C \exp (-k(\epsilon)|\alpha|)\|\psi\|^{2} .
$$

Next, we consider the real part of (2.24), which we write in the form

$$
\begin{aligned}
& \int_{0}^{1} \chi\left(\left|\psi^{\prime}\right|^{2}+\alpha^{2}|\psi|^{2}+\frac{U^{\prime \prime}\left(U-U\left(y_{c}\right)\right)}{|U-c|^{2}}|\psi|^{2}\right) d y \\
& =\int_{0}^{1} \frac{U^{\prime \prime}\left(c_{r}-U\left(y_{c}\right)\right)}{|U-c|^{2}} \chi|\psi|^{2}-\chi^{\prime} \operatorname{Re}\left(\psi^{\prime} \bar{\psi}\right) d y .
\end{aligned}
$$

We can now use (2.25) and (2.26) to bound the right-hand side by

$$
C\left(1+\frac{\left|c_{r}-U\left(y_{c}\right)\right|}{\left|c_{i}\right|}\right) \exp (-k(\epsilon)|\alpha|)\|\psi\|\left\|\psi^{\prime}\right\|
$$

By combining this result with (2.23), we conclude that we have a bound of the form

$$
\left\|\psi^{\prime}\right\|^{2}+\alpha^{2}\|\psi\|^{2} \leq \frac{\left|c_{r}-U\left(y_{c}\right)\right| \exp (-k(\epsilon)|\alpha|)}{\left|c_{i}\right|}\|\psi\|\left\|\psi^{\prime}\right\| .
$$

This is not possible if $\left|\alpha c_{i}\right| \gg\left|c_{r}-U\left(y_{c}\right)\right| \exp (-k(\epsilon)|\alpha|)$. 


\section{REFERENCES}

[1] P. G. Drazin and L. N. Howard, Hydrodynamic stability of parallel flow of inviscid fluid, Adv. Appl. Mech., 9 (1966), pp. 1-89.

[2] P. G. Drazin and W. H. Reid, Hydrodynamic Stability, Cambridge University Press, Cambridge, UK, 1981.

[3] S. Friedlander and A. Lipton-Lifschitz, Localized instabilities in fluids, in Handbook of Mathematical Fluid Dynamics 2, S. Friedlander and D. Serre, eds., North-Holland, Amsterdam, 2003, pp. 289-354.

[4] L. N. HowarD, The number of unstable modes in hydrodynamic stability problems, J. Mécanique, 3 (1964), pp. 433-443.

[5] J. L. Lions and E. Magenes, Non-Homogeneous Boundary Value Problems and Applications I, Springer, Berlin, 1972.

[6] W. Wasow, Asymptotic Expansions for Ordinary Differential Equations, Krieger, Huntington, 1976.

Copyright (c) by SIAM. Unauthorized reproduction of this article is prohibited. 